\title{
Definition and delivery of an aortopathy bundle of care (ABC): a tool for improving diagnosis and management of Marfan syndrome and related conditions
}

\author{
Authors: Emma Burkitt Wright, ${ }^{1,2}$ Sasha Henriques, ${ }^{1}$ Catherine Houghton, ${ }^{1}$ Bernard Clarke, ${ }^{2,3}$ Bernard Keavney ${ }^{2,3}$ \\ and Luigi Venetucci ${ }^{2,3}$
}

\section{Aims}

To accelerate diagnosis and improve management of Marfan syndrome and related conditions, a multidisciplinary improvement science project was developed between cardiology and genomic medicine.

\section{Methods}

Local experience suggested potential for improvement in the delivery of care for patients with Marfan syndrome and related conditions. Under the prevailing system, patients required separate clinical genetic and cardiological assessments. Delays in diagnosis were common and timely delivery of elements of follow-up, such as clinic appointments and imaging, was not always achieved. Current guidelines were reviewed to define key indices of effective management: the aortopathy bundle of care (ABC). A monthly genetic aortopathy team clinic (GATC) was established, permitting multidisciplinary consultations with a cardiologist, clinical geneticist and genetic counsellor. To optimise the utility of this new, resource-intensive clinic, plan-do-study-act cycles were embedded in each clinic, and increasing numbers of patients were seen each month. ABC measures were used to evaluate delivery of care using this model. Semi-structured questionnaires pre and post clinic were used to elicit patient feedback.

\section{Results}

Six elements to the $\mathrm{ABC}$ were defined: documentation of diagnosis according to revised Ghent criteria (and molecular genetic testing, where performed), clinical follow-up, genetic counselling provision, appropriate imaging, blood pressure management and appropriate referral for cardiac surgical opinion. Prior to the clinic's inception, concordance with the $\mathrm{ABC}$ was exceptionally low: no patients met all elements of this on retrospective review. Patients seen in the GATC received more appropriate and timely care in accordance with the ABC. The proportion of patients whose diagnosis was confirmed and documented according to revised Ghent criteria rose from $25 \%$ to $100 \% .100 \%$ of patients received appropriate imaging, compared to $25 \%$ previously. Improvements were also seen in hypertension management. Conclusions cannot yet be drawn on the ability of the GATC to provide reliably timed follow-up and appropriate cardiac surgical referral (due to the lead-time issue), and formal cost-effectiveness analysis will require these data. Patients reported high levels of satisfaction with the multidisciplinary approach. Clarification of diagnosis was possible in 11 patients from seven families, who were discharged from clinic as a result.

\section{Conclusions}

The ABC appears useful for measuring the effectiveness of care for patients with genetic aortopathies such as Marfan syndrome. The multidisciplinary GATC was shown to be effective in achieving a diagnosis in fewer clinic visits than previously, and provides a means of delivery of appropriate and comprehensive care to this patient group.

Authors: ${ }^{1}$ Manchester Centre for Genomic Medicine, Manchester, UK; ${ }^{2}$ University of Manchester, Manchester, UK; ${ }^{3}$ Manchester Heart Centre, Manchester, UK 